\title{
Radiation target volume definition after induction chemotherapy in locally advanced rectal cancer
}

\author{
Francesca De Felice $^{1} \cdot$ Daniela Musio $^{1} \cdot$ Vincenzo Tombolini $^{1,2}$
}

Accepted: 21 April 2016/Published online: 26 April 2016

(C) Springer-Verlag Berlin Heidelberg 2016

\section{Dear Editor:}

Neo-adjuvant treatment field design in locally advanced rectal cancer (LARC) is based on patterns of failure data. Considering that outcomes depend on both tumor $(\mathrm{T})$ and node $(\mathrm{N})$ stage, radiation therapy field arrangement is highly correlated with the primary tumor location as well as the areas and the distribution of lymph nodes judged to be at risk for local recurrence [1].

Generally, clinical target volume (CTV) includes the primary tumor site, the mesorectum (including mesorectal nodes), the presacral space (including the presacral nodes), and the lymph nodes along the internal iliac artery, in all patients. A high-dose CTV to the macroscopic tumor mass should be added. Treatment of obturator nodes is appropriate if primary tumor is within $10 \mathrm{~cm}$ from anal margin, whereas external iliac nodes are included if tumor directly invades anterior organs. Treatment of inguinal lymph nodes is indicated with extensive internal and external anal sphincter involvement by tumor or extension to the lower third of the vagina [2].

Nowadays, based on the efficacy demonstrated in colon cancer, the concept of induction chemotherapy has been developed in LARC, in order to reduce prin-

Francesca De Felice

fradefelice@hotmail.it

1 Department of Radiotherapy, Policlinico Umberto I "Sapienza" University of Rome, Viale Regina Elena 326, 00161 Rome, Italy

2 Spencer-Lorillard Foundation, Viale Regina Elena 262, Rome, Italy cipally distant metastasis incidence [3]. This poses a major contouring issue that needs considering. How should the target volume in responder patients who had positive regional lymph nodes, as well as organ invasion at diagnosis, be planned? Contouring recommendations are lacking on this topic. Probably, considering the consolidate trimodality approach in LARC and thus the certain subsequent surgery, modification of target volume definition following IC could be considered. On the other hand, contouring pre-IC disease could guarantee a higher percentage of clinical complete response, hazarding a more conservative surgical management, such as endoluminal loco-regional resection by transanal endoscopic microsurgery (TEM).

In the induction chemotherapy era, a proper definition of radiation target volumes is paramount to reduce local recurrence and improve patient quality of life. The value of both target volume proposal should be addressed in a definitive randomized trial. The need of definitive contouring guidelines, in this setting of patients, will potentially increase in the near future.

\section{References}

1. National Comprehensive Cancer Network Guidelines, Rectal Cancer, Version 3.2015. www.ncen.org

2. Roels S, Duthoy W, Haustermans K, Penninckx F, Vandecaveye V, Boterberg T, De Neve W (2006) Definition and delineation of the clinical target volume for rectal cancer. Int J Radiat Oncol Biol Phys 65(4):1129-42

3. De Felice F, Musio D, Tombolini V (2016) Does the addition of induction chemotherapy improve out come in locally advanced rectal cancer? Int J Color Dis 31(3):789 